\title{
DUSTY DISKS AROUND CENTRAL STARS OF PLANETARY NEBULAE
}

\author{
Geoffrey C. Clayton ${ }^{1}$, Orsola De Marco $^{2,3}$, Jason Nordhaus ${ }^{4,8}$, Joel Green $^{5}$, \\ Thomas Rauch ${ }^{6}$, Klaus Werner ${ }^{6}$, and You-Hua $\mathrm{CHU}^{7}$ \\ ${ }^{1}$ Department of Physics \& Astronomy, Louisiana State University, Baton Rouge, LA 70803, USA; gclayton@fenway.phys.1su.edu \\ ${ }^{2}$ Department of Physics \& Astronomy, Macquarie University, Sydney, NSW 2109, Australia; orsola@ science.mq.edu.au \\ ${ }^{3}$ Astronomy, Astrophysics, and Astrophotonics Research Centre, Macquarie University, Sydney, NSW 2109, Australia \\ ${ }^{4}$ Center for Computational Relativity and Gravitation, and National Technical Institute for the Deaf, \\ Rochester Institute of Technology, Rochester, NY 14623, USA; nordhaus@ astro.rit.edu \\ ${ }^{5}$ Department of Astronomy, The University of Texas, 1 University Station, C1400, \\ Austin, TX 78712-0259, USA; joel@astro.as.utexas.edu \\ ${ }^{6}$ Institute for Astronomy \& Astrophysics, Kepler Center for Astro and Particle Physics, Eberhard Karls University, \\ Sand 1, D-72076 Tübingen, Germany; rauch@astro.uni-tuebingen.de, werner@astro.uni-tuebingen.de \\ ${ }^{7}$ Department of Astronomy, University of Illinois at Urbana-Champaign, \\ 1002 West Green Street, Urbana, IL 61801, USA; chu@ astro.uiuc.edu \\ Received 2013 August 31; accepted 2014 March 19; published 2014 May 6
}

\begin{abstract}
Only a few percent of cool, old white dwarfs (WDs) have infrared excesses interpreted as originating in small hot disks due to the infall and destruction of single asteroids that come within the star's Roche limit. Infrared excesses at $24 \mu \mathrm{m}$ were also found to derive from the immediate vicinity of younger, hot WDs, most of which are still central stars of planetary nebulae (CSPNe). The incidence of CSPNe with this excess is $18 \%$. The Helix CSPN, with a $24 \mu \mathrm{m}$ excess, has been suggested to have a disk formed from collisions of Kuiper belt-like objects (KBOs). In this paper, we have analyzed an additional sample of CSPNe to look for similar infrared excesses. These CSPNe are all members of the PG 1159 class and were chosen because their immediate progenitors are known to often have dusty environments consistent with large dusty disks. We find that, overall, PG 1159 stars do not present such disks more often than other CSPNe, although the statistics (five objects) are poor. We then consider the entire sample of CSPNe with infrared excesses and compare it to the infrared properties of old WDs, as well as cooler post-asymptotic giant branch (AGB) stars. We conclude with the suggestion that the infrared properties of CSPNe more plausibly derive from AGB-formed disks rather than disks formed via the collision of KBOs, although the latter scenario cannot be ruled out. Finally, there seems to be an association between CSPNe with a $24 \mu \mathrm{m}$ excess and confirmed or possible binarity of the central star.
\end{abstract}

Key words: circumstellar matter - planetary nebulae: general - stars: evolution - white dwarfs

Online-only material: color figures

\section{INTRODUCTION}

There are three indicators of planetary debris around white dwarfs (WDs): (1) metal pollution, discovered decades ago (e.g., Weidemann 1960), but originally thought to be accretion from the interstellar medium (ISM; e.g., Aannestad et al. 1993; Dupuis et al. 1993); (2) an infrared (IR) excess due to warm dust and first detected around G29-38 (Zuckerman \& Becklin 1987); and (3) gaseous disks (e.g., Gänsicke et al. 2006), which unambiguously showed that the debris material is an accretion disk lying within the tidal disruption radius of the WD (Reach et al. 2005). The debris material comes from the disruption of asteroids (Graham et al. 1990; Jura 2003). These three indicators of debris accretion are hierarchical. All WDs that have gaseous disks also have dust/IR excesses, and all WDs that have dust also have metal-polluted atmospheres (Brinkworth et al. 2009, 2012). However, this does not work in the opposite direction, i.e., not all metal-polluted WDs have dust, and not all dusty WDs have gas. The fraction of cool WDs with IR excesses is 1-3\% (Farihi et al. 2009; Steele et al. 2011; Girven et al. 2011; Barber et al. 2012). These disks are typically found very close $\left(<1 R_{\odot}\right)$ to cool $(<25,000 \mathrm{~K})$ WDs. The dust in these disks is quite warm $(\sim 1000 \mathrm{~K})$, and so the dust emission shows up strongly in the Spitzer IRAC bands (Xu \& Jura 2012). Many questions still remain about the structure and longevity of these

\footnotetext{
8 NSF Fellow.
}

disks and what they are telling us about the planetary systems that once orbited these stars, but other than that, the debris nature of these disks seems to be a reasonable interpretation.

In 2007, a dust disk was detected around the central star of the Helix planetary nebula (PN), also known as NGC 7293 (Su et al. 2007). This disk differed greatly from those previously found around WDs. In the Helix system, the star is much hotter $(110,000 \mathrm{~K}$ versus $<25,000 \mathrm{~K}$ of the typical WD with debris disks), and the dust is much colder $(\sim 100 \mathrm{~K}$ versus $1000 \mathrm{~K}$ for the WD disks) and lies much farther from the star ( $\sim 50 \mathrm{AU}$ versus $<0.01$ AU for the WD disks; Su et al. 2007; Bilíková et al. 2012). Nonetheless, this object was also interpreted as having a debris disk, but the suggested origin was dust production by collisions among Kuiper belt-like objects (KBOs), rather than individual asteroids pulverized by entering the Roche limit.

Further surveys have found cold dust disks around a number of hot WDs and central stars of PNe (CSPNe). Chu et al. (2011) looked at 71 stars and found 9 disks. Seven of these nine stars with disks were CSPNe. The other two are hot WDs likely to have been PNe in the very recent past (Tweedy \& Kwitter 1994; Werner et al. 1997). Out of the $35 \mathrm{CSPNe}$ analyzed by Chu et al. (2011), 7 detections represent a detection rate of $20 \%$. Bilíková et al. (2012) searched an additional sample of CSPNe. Out of 56 viable candidates, they detected disks in 17-20\% of their sample. For the combined samples, the incidence of disks is $18 \%$. The much higher frequency of disks around CSPNe than around cool WDs raised questions as to whether their 
Table 1

PG 1159 Stars with PNe Observed with Spitzer ${ }^{\mathrm{a}}$

\begin{tabular}{|c|c|c|c|c|c|}
\hline PN Name & Star Name & $\begin{array}{c}T_{\text {eff }} \\
(\mathrm{kK})\end{array}$ & $\begin{array}{c}d \\
(\mathrm{pc})\end{array}$ & IRAC $^{b}$ & $\begin{array}{c}\text { MIPS } / 24^{\mathrm{c}} \\
(\mathrm{mJy})\end{array}$ \\
\hline NGC $246^{\mathrm{d}, \mathrm{e}}$ & WD 0044-121 & 150 & 495 & Yes & $<26.2$ \\
\hline NGC $650^{d}$ & WD $0139+513$ & 140 & 746 & Yes & $<30$ \\
\hline StWr 3-2 & Wray $17-1^{\mathrm{d}}$ & 140 & $\cdots$ & $\cdots$ & $\cdots$ \\
\hline $\mathrm{A} 21^{\mathrm{e}}$ & WD $0726+133$ & 140 & 541 & Yes & $0.916 \pm 0.114$ \\
\hline $\operatorname{JnEr} 1^{\mathrm{d}, \mathrm{e}}$ & WD $0753+535$ & 130 & 1145 & Yes & $<0.675$ \\
\hline $\operatorname{Lo} 4^{\mathrm{d}, \mathrm{e}}$ & WD $1003-441$ & 120 & 3000 & $\ldots$ & $<19.9$ \\
\hline $\mathrm{A} 43^{\mathrm{d}, \mathrm{e}}$ & WD $1751+106$ & 110 & 2050 & $\ldots$ & $<6.1$ \\
\hline NGC $6765^{\mathrm{d}}$ & WD 1909+304 & $\cdots$ & 2334 & $\cdots$ & $\cdots$ \\
\hline NGC $6852^{\mathrm{d}, \mathrm{e}}$ & WD 1958+015 & $\cdots$ & 2736 & $\cdots$ & $<140.4$ \\
\hline NGC $7094^{\mathrm{d}}$ & WD $2134+125$ & 110 & 1390 & $\ldots$ & $\ldots$ \\
\hline Jn $1^{\mathrm{e}}$ & WD $2333+301$ & 170 & & $\ldots$ & $<0.824$ \\
\hline Jacoby 1 & WD $1520+525^{\mathrm{e}}$ & 150 & 600 & $\cdots$ & $<0.358$ \\
\hline MWP $1^{\mathrm{e}}$ & WD $2115+339$ & 170 & 1400 & $\cdots$ & $<0.903$ \\
\hline
\end{tabular}

Notes.

${ }^{\text {a }}$ From http://www.arm.ac.uk/ csj/research/pg1159catalog.html.

b IRAC imaging is available.

${ }^{\mathrm{c}}$ MIPS $24 \mu \mathrm{m}$ flux estimates are from Chu et al. (2011) except for NGC 650 which is from this study.

d This study.

e Chu et al. (2011), Bilíková et al. (2012).

nature is also that of debris disks or whether these disks are formed by mass loss from the stars during the asymptotic giant branch (AGB) phase. Dusty outflows, disks, and shells are, of course, known and expected to be associated with cool AGB and post-AGB stars, but one might expect them to be destroyed by the heating of their central stars. The presence and longevity of disks around CSPNe and young, hot WDs can inform us about the recent past of these stars, including the presence of a binary companion that may have influenced the AGB mass-loss process, as well as about dust formation and survival.

These questions led us to look for more disks around CSPNe. We selected the PG 1159 stars that constitute about 10\%-20\% of the entire CSPN group. These stars are intermediate between the Wolf-Rayet CSPNe (also known as the [WC] stars) and WDs. The reason for targeting these stars is that some of their immediate progenitors, the [WC] stars, are known to have spectacular dusty environments with large silicate disks, as well as carbon-rich outflows (e.g., CPD -56 8032; Cohen et al. 1999). ${ }^{9}$ Abundance measurements of debris around cool, metal-polluted WDs imply that the material is very carbon-poor material (e.g., Gänsicke et al. 2012; Jura et al. 2012). PG 1159 stars follow the [WC] central stars by no more than $10^{3}-10^{4} \mathrm{yr}$ (using a stellar evolutionary track for a $0.60-0.63 M_{\odot}$ star from Vassiliadis \& Wood 1993 and known PG 1159 stellar parameters from Dreizler \& Heber 1998), while the oldest PG 1159 stars, those with no PNe, are another $10^{4}-10^{5}$ yr older than that. Since the dust around Wolf-Rayet central stars may be distributed in long-lived Keplerian disks, it is possible that these disks have survived the hottest phases of the star and are present in the PG 1159 stage.

9 All known examples of oxygen-carbon chemistry in [WC] stars are in the [WCL] ("L" for late) class, in the immediate post-AGB phase, with temperatures of 30-50 kK. Although there have been claims that at least one of the hotter, earlier [WC] objects also shows the dual-dust signature of a disk, we note that the only claim of dual-dust for a [WCE] central star (NGC 5315) is by De Marco \& Soker (2002), cited in a private communication. A similar claim was made by Perea-Calderón et al. (2009), with no citation. We examined the Infrared Space Observatory (ISO) Short Wavelength Spectrometer spectrum of NGC 5315, and there is no sign of silicate features. The ISO spectrum was also classified as 4/5.Eu: by Hodge et al. (2004), which indicates atomic and polycyclic aromatic hydrocarbon emission but no silicate features.
We have therefore obtained Spitzer/Infrared Spectrograph (IRS) spectra of a sample of nine PG 1159 stars, all associated with $\mathrm{PNe}$, to look for evidence of dusty disks, increase the statistics of disks around CSPNe, compare them further with debris disks around old WDs, as well as with that around the Helix CSPN, and study the disk longevity around CSPNe.

\section{OBSERVATIONS AND REDUCTION}

Nine PG 1159 stars, listed in Table 1, were observed with the Spitzer/IRS (Houck et al. 2004). Staring-mode observations took place during IRS campaigns 58.1-58.3, 59.2, and 61.1 during 2008 December 5-9 and 2009 January 9-25, March 5-8, and April 29. All objects were observed with the short-low (SL; 5.2-14 $\mu \mathrm{m} ; \lambda / \Delta \lambda \sim 90$ ) and long-low (LL; 14-38 $\mu \mathrm{m}$; $\lambda / \Delta \lambda \sim 90)$ low-resolution modules. The LL slit is $168^{\prime \prime} \times 10^{\prime \prime} .7$, and the SL slit is $57^{\prime \prime} \times 33^{\prime \prime} 7$.

Our point-source extraction and calibration method has been extensively detailed previously (Furlan et al. 2006; Sargent et al. 2006; Watson et al. 2007; Sargent et al. 2009). We start with the Spitzer Science Center IRS pipeline Basic Calibrated Data (BCD) product for each object. The BCD is flat fielded, dark current subtracted, and stray light corrected. We employ a point-source spectral extraction and calibration method using the Spectral Modeling, Analysis, and Reduction Tool (SMART; Higdon et al. 2004) and IDL routines for post-pipeline processing. In particular, we identify and correct for rogue $(\mathrm{NaN})$ pixels in our two-dimensional spectral data by linearly interpolating the four nearest neighboring pixel values. To correct for the sky background, we subtract the off-order spectrum $\left(\sim 1^{\prime}-3^{\prime}\right.$ away from target) in the same nod position of the on-target order. The low-resolution, sky-subtracted spectra are then extracted using a variable-width extraction window that fits tightly to the IRS point-spread function.

To calibrate our spectra, we employ custom relative spectral response functions (RSRFs) which yield flux densities based on the signal detected at a given wavelength. To produce the RSRFs, we use SMART to divide each of our spectra, nod by nod, for each order of each module. A spectral template of a calibration star ( $\alpha$ Lac; Cohen et al. 2003) was identically 


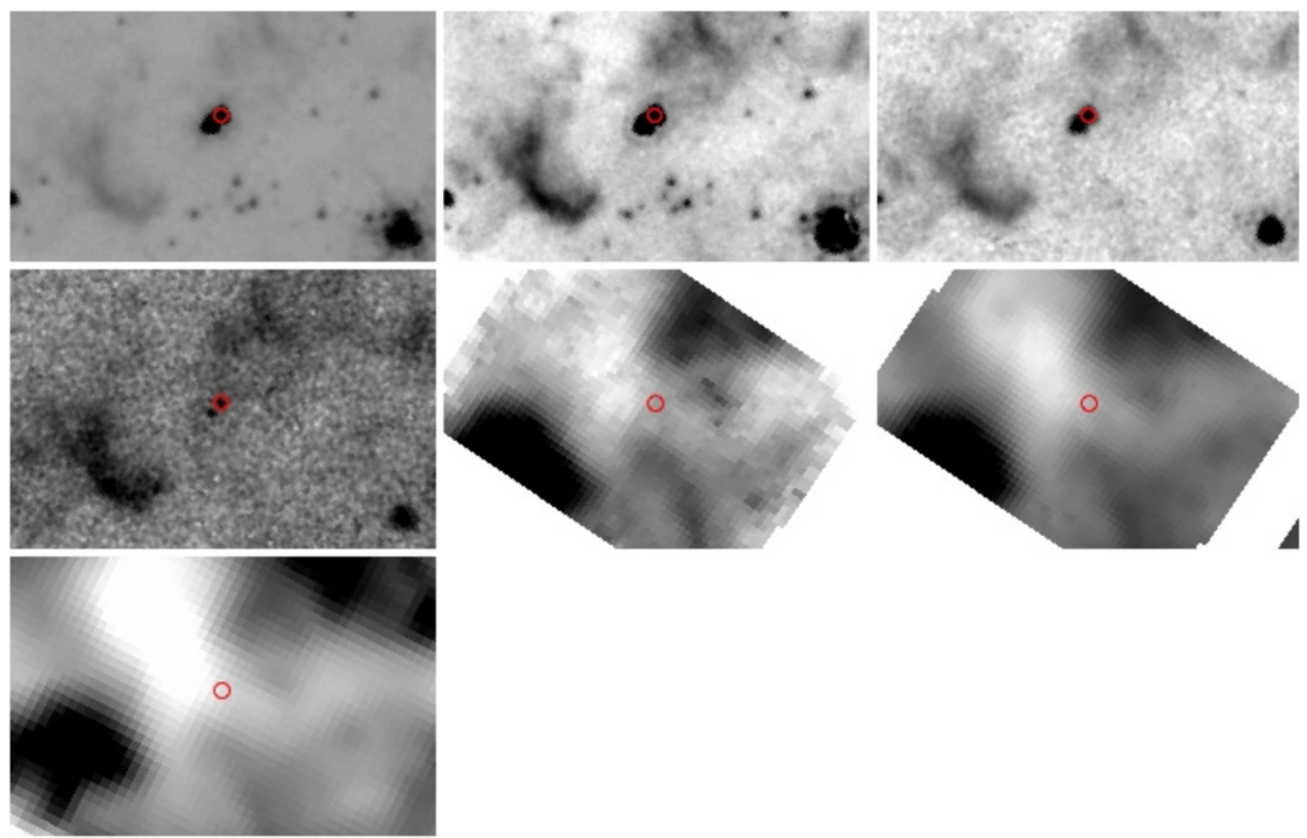

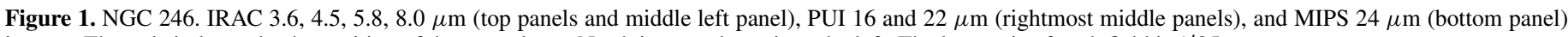
images. The red circle marks the position of the central star. North is up and east is to the left. The long axis of each field is 1 '.85.

(A color version of this figure is available in the online journal.)

prepared such that the quotient of the template and the observed stellar spectrum is the RSRF. The low-resolution, sky-subtracted extractions were then multiplied by the RSRF, corresponding to the relevant nod, order, and module. In general, we found good agreement between flux values in regions of wavelength overlap between orders in each module. For each object, the procedure described above produces a calibrated point-source spectrum.

There are IRS/Peakup images (PUI; 16 and $22 \mu \mathrm{m}$ ) for the whole sample. In addition, three stars have IRAC (3.6, 4.5, 5.8, $8.0 \mu \mathrm{m})$ images, and six have MIPS $(24 \mu \mathrm{m})$ images (e.g., Chu et al. 2011). See Table 1 for a summary. These images have been examined for point sources at the coordinates of the sample stars. As an example, Figure 1 shows existing IR images of NGC 246. NGC 246 and NGC 650 show a point source at the position of the star in all four IRAC bands. The images also clearly show the nearby companions of these two stars. JnEr 1 shows a point source only at 3.6 and $4.5 \mu \mathrm{m}$. None of the stars with MIPS $24 \mu \mathrm{m}$ imaging shows a point source at the location of the star. Also, there are no detections of point sources in any of the PUI images for the nine stars in the sample.

The IRS spectra for the sample stars are plotted in Figure 2. These spectra show strong emission lines due to the surrounding PNe (Guiles et al. 2007). The primary lines are [S IV] $\lambda 10.51$, [Ne v] $\lambda 14.32,[\mathrm{Ne}$ III] $\lambda 15.55,[\mathrm{~S} \mathrm{III]} \lambda 18.71,[\mathrm{Ne} \mathrm{v}] \lambda 24.30$, [O IV] $\lambda 25.89$, and [S III] $\lambda 33.47 \mu \mathrm{m}$. A point-source extraction was attempted for both slits for each star, which are shown in Figure 2. The sky subtraction is taken $\sim 1^{\prime}-3^{\prime}$ away from the star, well outside the slit position and outside the visible PN nebulosity. So the one-dimensional spectra, extracted and shown in Figure 2, include any diffuse dust emission from the PNe present in the portions of the slit that were extracted. The two-dimensional spectra have point-like sources in the slit that have been extracted, but in each case these turned out to be dust condensations in the PNe. Local sky subtraction was also attempted, but no significant point sources centered on the target stars were detected. As seen in Figure 2, all of the stars in the sample show mid-IR emission rising toward longer wavelengths, indicating the presence of cool dust. The key question is, where is the dust whose emission is being detected? Is the IR emission from a point source centered on the star, in which case it would be consistent with dust emission from a circumstellar disk, or is it diffuse emission from dust associated with the PN that surrounds each of these stars?

Spectral energy distributions (SEDs) were constructed for the three stars, NGC 246, NGC 650, and JnEr 1, with both IRAC and MIPS $24 \mu \mathrm{m}$ photometry. SEDs for each of the three stars are shown in Figure 3, where the fluxes were all normalized to the distance of NGC 246 (500 pc, Bond \& Ciardullo 1999). For the IRAC images, which overlap in wavelength with the IRS spectra, the brightness of the star is plotted both with sky subtraction (in an annulus close to the star) and with no sky subtraction. Since none of the stars showed a point source at $24 \mu \mathrm{m}$, only the no-sky-subtraction $24 \mu \mathrm{m}$ fluxes are plotted in Figure 3. The no-sky-subtraction fluxes closely mimic the fluxes of the IRS extractions. This clearly shows two important results. First, the sky-subtracted fluxes, in all three cases, are consistent with the tail of the blackbody SED from the star. Second, the no-sky-subtraction fluxes agree very well with the IRS fluxes. Therefore, the conclusion must be that the mid-IR emission from dust seen in the IRS spectra comes from diffuse emission in the PNe surrounding each of the PG 1159 stars. This is reinforced by the fact that the six stars in our sample, which were also imaged with MIPS at $24 \mu \mathrm{m}$, show no evidence for a point source. Therefore, we see no evidence for dusty disks in this small sample of PG 1159 stars.

In total, including our sample, 20 PG 1159 stars (10 with $\mathrm{PNe}$ and 10 without) from the catalog of C. S. Jeffery ${ }^{10}$ have been observed with Spitzer/MIPS at $24 \mu \mathrm{m}$. None of the PG 1159 stars with no PNe, including PG 1159-035 itself, shows evidence for a dusty disk (Chu et al. 2011). The 10 stars with $\mathrm{PNe}$ are listed in Table 1.

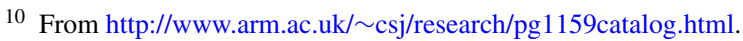



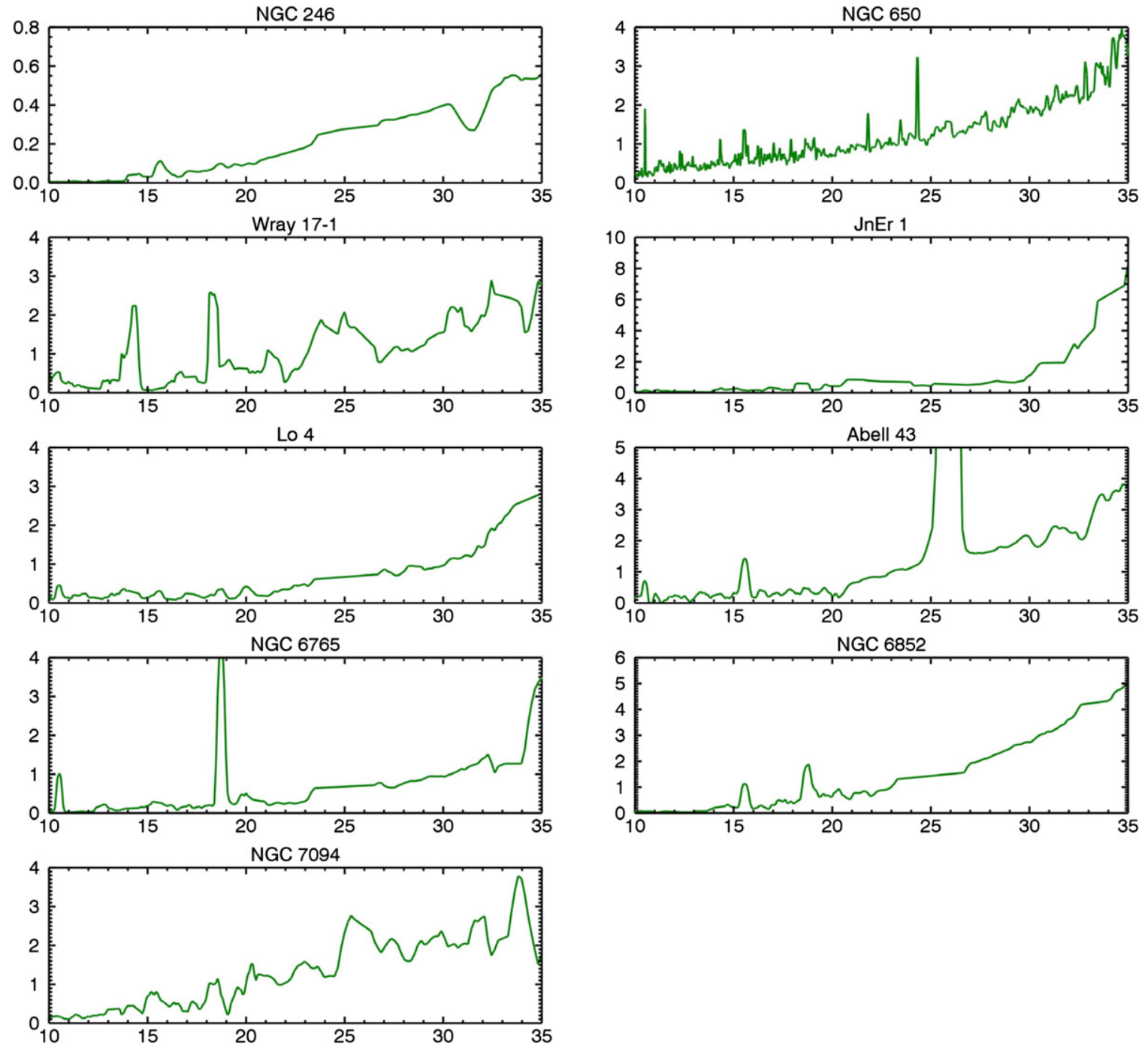

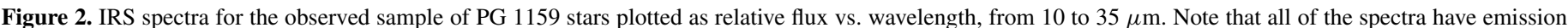

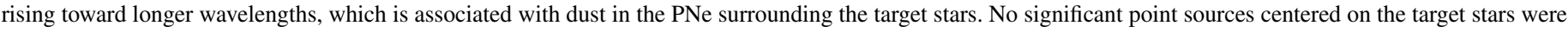
detected.

(A color version of this figure is available in the online journal.)

There is Two Micron All Sky Survey (2MASS) JHK photometry available for NGC 246 and NGC 650. UBVRI photometry was available for NGC 246 (Kilkenny et al. 1998), and Sloan Digital Sky Survey ugriz photometry is available for NGC 650 and JnEr 1 (Abazajian et al. 2009). Low-resolution IUE spectra were downloaded from the MAST archive for NGC 246. Photometry of NGC 246 and NGC 650 is difficult because each has close companions. NGC 246 has a physical G8-K0 V companion 3".8 away (Bond \& Ciardullo 1999), and NGC 650 has two stars, thought to be background objects, only 1".3 away (Koornneef \& Pottasch 1998).

\section{THE PG 1159 CSPN IN THE CONTEXT OF OTHER CSPNe}

The [WC] CSPNe are thought to evolve to be PG 1159 stars with a PN, then PG 1159 stars without a PN, and finally non-DA WDs (Werner \& Herwig 2006). Dusty disks may be common around $[\mathrm{WC}]$ central stars because they often exhibit dual-dust chemistry (the simultaneous presence of the carbonrich polycyclic aromatic hydrocarbons and oxygen-rich silicate dust; e.g., De Marco \& Soker 2002), a phenomenon that has been attributed to the presence of an oxygen-rich disk coexisting alongside a carbon-rich outflow (e.g., Cohen et al. 1999). The fraction of disks among the [WC] central stars may be difficult to find using an infrared-excess technique due to the particularly red colors of the hydrogen-deficient atmospheres of these stars. However, if present, they should be readily detected around the progeny of the [WC] stars, the PG 1159 stars.

For CSPNe, in general, $18 \%$ of 84 objects were detected to have a $24 \mu \mathrm{m}$ flux excess (Bilíková et al. 2012). Of the ten PG 1159 stars with PNe observed at $24 \mu \mathrm{m}$, five have such low signal-to-noise ratio that they do not constrain the presence of a disk. One of the remaining five, the central star of the PN A21, has been detected at $24 \mu \mathrm{m}$ (Chu et al. 2011). Therefore, for this small sample, the detection rate of one in five objects is fully in line with the statistics of CSPNe. The fact that we did not detect a preponderance of circumstellar disks among the PG 1159 stars argues either in favor of their disks having been destroyed by intense radiation, that not all [WC] stars and, therefore, their descendant PG 1159 stars, have such disks, or that we are just observing the effect of low number statistics. 


\section{THE CLASSIFICATION OF STARS WITH DISKS}

Before we discuss whether disks have a debris origin or not, we should clarify how the host stars should be classified. We have seen $24 \mu \mathrm{m}$ excesses in WDs, both cool (old) and hot (young), and in CSPNe. CSPNe have very recently departed from the AGB and are at a similar evolutionary stage as the warm WDs. The cool WDs, on the other hand, left the AGB a long time ago.

There is a second source of confusion. Some objects classified as PNe were later found to be mimics (Frew 2008; Frew \& Parker 2010). There are two types of mimics: young, hot WDs that lost their PNe some time ago, but are still hot and luminous enough to ionize the ISM, and stars that, by their temperature and gravity, can be placed on post-red giant branch (RGB) evolutionary tracks. If hot enough, these stars can also ionize the ISM and have a nebula that can be mistaken for a PN. An example of the former class is the disk object, EGB 1. Its nebulosity is not a bona fide PN, but is ionized ISM around a young, hot WD; the central star of EGB 1 likely was surrounded by a PN until recently. Another similar mimic is the nebulosity discovered around the hot WD 0109+111 by Werner et al. (1997). Both EGB 1 and WD 0109+111 should be analyzed in the context of CSPNe when it comes to interpreting their disks.

The latter class of mimics constitutes a third category of objects. An example is the disk object DeHt 5, which is traditionally listed as a PN (Frew 2008; De Marco et al. 2013). The central star's temperature and gravity place this star very close to the post-RGB track of a $\sim 0.44 M_{\odot}$ star (Napiwotzki 1999), although Gianninas et al. (2010) find a mass of $\sim 0.54 M_{\odot}$, which is more consistent with a post-AGB origin. As a mimic, this object is similar to EGB 5 and PHL 932 (Geier et al. 2011, neither of which is known to have a disk). De Marco et al. (2013) detected a $4 \sigma J$-band excess for the central star of DeHt 5, consistent with an M5V companion. Bilíková et al. (2012) detected point sources in 2MASS, IRAC, and MIPS bands with a clear excess at 8 and $24 \mu \mathrm{m}$. They concluded that there is a cool disk with a temperature of $190 \mathrm{~K}$ and a radius of $8.2 \mathrm{AU}$. Whatever the origin of this disk (most likely a stellar interaction that happened while the star was on the RGB, possibly a common envelope if the companion is found to orbit the star closely), Bannister et al. (2003) examined HST/ STIS UV spectra and found hot, circumstellar gas, with lines redshifted relative to the WD rest frame, and speculate that this might indicate infalling matter. This object is a third category of star with a dusty circumstellar environment alongside bona fide old WDs and younger, warmer WDs with or without a PN.

Finally, we introduce the post-AGB stars. These are F or G supergiants that have departed the AGB, but are still much cooler than CSPNe. These stars can have a pre-PN (a nebula that shines because of reflected light), in which case they are called central stars of pre-PN, or not, in which case we call them naked post-AGB stars (Bright et al. 2012).

\section{DO ANY OF THE CSPN DISKS HAVE A DEBRIS NATURE?}

The last question we want to address is whether any of the disks found around CSPNe are debris disks. While we acknowledge that CSPN debris disks cannot be like those around old, cool WDs, we wonder whether other CSPN disks could be formed by collisions of KBOs, as was suggested for the Helix PN by $\mathrm{Su}$ et al. (2007). That scenario is plausible in the case of the Helix PN as investigated analytically by Dong
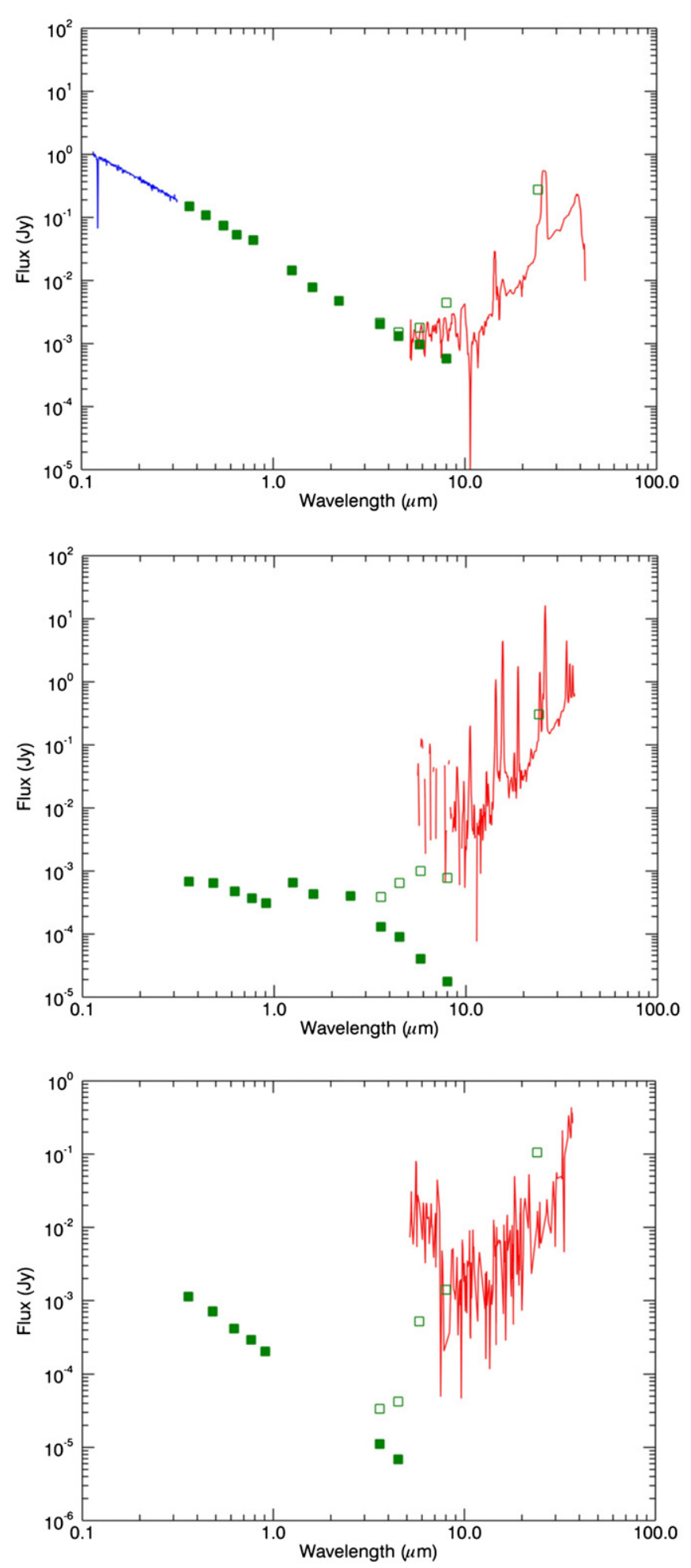

Figure 3. SEDs for NGC 246 (top panel), NGC 650 (middle panel), and JnEr 1 (bottom panel). The IUE spectrum is in blue and IRS spectra are in red. The solid green squares denote the sky-subtracted photometry in the $U, B, V, R, I, J, H$, and $K$ bands and IRAC bands at 3.6, 4.5, 5.8, and $8.0 \mu \mathrm{m}$. The open squares denote the photometry without sky subtraction. The MIPS $24 \mu \mathrm{m}$ point is included. The close companions to NGC 650 are contaminating the photometry in the $J$, $H$, and $K$ bands.

(A color version of this figure is available in the online journal.)

et al. (2010). Disks around CSPN can have formed from gas ejected during the AGB, plausibly, but not necessarily, because of a binary interaction, within which dust condensed. This is the 
Table 2

Stars for [8] versus [8-24] Plot

\begin{tabular}{|c|c|c|c|c|c|c|c|}
\hline Star Name & Nebula Name & Star Type & $\begin{array}{c}T_{\text {eff }} \\
(\mathrm{kK})\end{array}$ & $\begin{array}{c}d \\
(\mathrm{pc})\end{array}$ & $\begin{array}{c}{[8]^{\mathrm{a}}} \\
(\mathrm{mag})\end{array}$ & $\begin{array}{r}{[8-24]^{\mathrm{a}}} \\
(\mathrm{mag})\end{array}$ & Ref. $^{b}$ \\
\hline GD 133 & $\ldots$ & Old WD & 12.2 & 43 & 18.18 & 1.95 & $2, \mathrm{C}$ \\
\hline GD 56 & $\ldots$ & Old WD & 14.4 & 74 & 16.05 & 0.72 & $2, \mathrm{C}$ \\
\hline WD $2326+049$ & $\ldots$ & Old WD & 11.8 & 14 & 17.50 & 1.05 & $7, \mathrm{~K}$ \\
\hline WD $0146+187$ & $\ldots$ & Old WD & 11.5 & 48 & 17.98 & 0.95 & $8, \mathrm{~L}$ \\
\hline WD $2115-560$ & $\ldots$ & Old WD & 9.7 & 22 & 18.94 & 0.93 & $8, \mathrm{~L}$ \\
\hline WD $1729+371$ & $\ldots$ & Old WD & 10.5 & 57 & 17.21 & 1.22 & $9, \mathrm{~L}$ \\
\hline WD $0726+133$ & A21 & CSPN, PG 1159 & 140 & 540 & $>15.64$ & $>6.08$ & $1, \mathrm{~A}$ \\
\hline \multirow[t]{2}{*}{ WD 0044-121 } & NGC 246 & CSPN, PG $1159^{c}$ & 150 & 500 & 12.74 & $<6.65$ & $1, \mathrm{~B}$ \\
\hline & Helix & $\operatorname{CSPN}^{\mathrm{d}}$ & 110 & 215 & 15.74 & 8.49 & $1,3, \mathrm{D}$ \\
\hline WD $1957+225$ & NGC 6853 & $\mathrm{CSPN}^{\mathrm{e}}$ & 130 & 379 & 12.95 & 6.12 & $1, \mathrm{E}$ \\
\hline HD 293373 & NGC 2346 & $\mathrm{CSPN}^{\mathrm{f}}$ & 100 & 900 & 4.36 & 1.58 & $1, \mathrm{~F}$ \\
\hline HD 183932 & NGC 6804 & $\mathrm{CSPN}^{\mathrm{g}}$ & 85 & 1470 & 4.51 & 2.79 & $1, \mathrm{G}$ \\
\hline WD $0103+732$ & EGB 1 & Young WD; CSPN mimic ${ }^{\mathrm{e}}$ & 147 & 650 & 13.65 & 5.68 & $1, \mathrm{~J}$ \\
\hline \multirow[t]{2}{*}{ WD 0439+466 } & Sh 2-216 & CSPN & 95 & 129 & 16.82 & 6.65 & $1, \mathrm{~J}$ \\
\hline & K 1-22 & $\mathrm{CSPN}^{\mathrm{c}}$ & 141 & 1330 & 10.12 & 2.69 & $1, \mathrm{~J}$ \\
\hline \multirow[t]{9}{*}{ WD 0950+139 } & EGB 6 & $\mathrm{CSPN}^{\mathrm{c}}$ & 108 & 645 & 10.02 & 3.62 & $1, \mathrm{~J}$ \\
\hline & NGC 7139 & CSPN & 117 & 2400 & 10.27 & $\cdots$ & $1, \mathrm{D}$ \\
\hline & NGC 2438 & $\mathrm{CSPN}^{\mathrm{h}}$ & 114 & 1200 & 12.54 & 7.54 & $1, \mathrm{~J}$ \\
\hline & IRAS $16279-4757^{\mathrm{i}}$ & Pre-PN & 5.7 & 2000 & -1.87 & 4.48 & $4, \mathrm{H}$ \\
\hline & IRAS 08005-2356 ${ }^{\mathrm{i}}$ & Pre-PN ${ }^{\mathrm{j}}$ & 6.8 & 4000 & -2.84 & 3.13 & $5, \mathrm{I}$ \\
\hline & Average O-rich & Post-AGB & $\ldots$ & 50,000 & -1.50 & 2.50 & 6 \\
\hline & Average C-rich & Post-AGB & $\ldots$ & 50,000 & -2.50 & 4.50 & 6 \\
\hline & Average C-rich & Post-AGB & $\ldots$ & 50,000 & -2.50 & 4.50 & 6 \\
\hline & DeHt $5^{\mathrm{k}}$ & Post-RGB ${ }^{\mathrm{e}}$ & 70 & 345 & 15.51 & 5.56 & $1, \mathrm{D}$ \\
\hline
\end{tabular}

\section{Notes.}

a All fluxes have been scaled to $500 \mathrm{pc}$ and converted to magnitudes.

b Flux references: (1) Bilíková et al. 2012; (2) Jura et al. 2007a; (3) Su et al. 2007; (4) Matsuura et al. 2004; (5) Slijkhuis et al. 1991; (6) Woods et al. 2011; (7) Reach et al. 2005; (8) Farihi et al. 2009; (9) Jura et al. 2007 b.

$T_{\text {eff }}$ references: (A) Werner et al. 2004; (B) Koesterke 2001; (C) Jura et al. 2007a; (D) Frew 2008; (E) Napiwotzki 1999; (F) Mendez \& Niemela 1981; (G) Phillips 2003; (H) Hu et al. 1993; (I) Slijkhuis et al. 1991; (J) Bilíková et al. 2012; (K) Reach et al. 2005 ; (L) Farihi et al. 2009.

c Wide binaries Ciardullo et al. 1999; Liebert et al. 2013.

d No companion brighter than mid-to-late M (Su et al. 2007).

e $I$ - and $J$-band excess binary candidates (De Marco et al. 2013).

${ }^{f}$ Close binary (Mendez \& Niemela 1981).

g Suspected binary (Frew 2008).

h Infrared excess binary candidate (Bilíková et al. 2012).

${ }^{\mathrm{i}}$ Used MSX A and E fluxes instead of IRAC 8 and $24 \mu \mathrm{m}$.

j Suspected binary (S. N. Bright et al. 2014, in preparation).

k PN mimic; see Section 4.

likely origin of disks around naked post-AGB stars (van Winckel et al. 2009), central stars of pre-PN (e.g., Bright et al. 2012), as well as disks around some CSPN (e.g., De Marco et al. 2002). Both scenarios have been amply discussed by Bilíková et al. (2012), who point out the impossibility of discerning between them. Here, we examine the possibility that the Helix disk, as well as those detected around other PN central stars, have more plausibly an AGB origin than a shattered KBO one.

In Figure 4, we present a color-magnitude diagram of all the objects for which 8 and/or $24 \mu \mathrm{m}$ imagery exists. All fluxes have been scaled to $500 \mathrm{pc}$ using the distances listed in Table 2. The cool, old WDs have dim hot disks, as expected. The CSPNe with data, on the other hand, reside in a strip extending from the top left to the bottom right of the figure, with the brightest members being much brighter, but only slightly redder than the cool WDs, and the dimmest members being only slightly brighter, but far redder than the cool WDs. The two brightest CSPN objects, NGC 2346 and NGC 6804, are known from independent observations to have large dusty tori: the former, a bright edge-on, bipolar PN, has photometric variability due to the close binary companion periodically hiding behind dust clumps in the disk (Kato et al. 2001 and references therein), while the latter has a possibly crystalline silicate feature in a Gemini/Michelle spectrum (Bilíková et al. 2012), indicating a long-lived Keplerian disk. The PG 1159 stars, A 21, and NGC 246 have only limits, and while the former has a $24 \mu \mathrm{m}$ limit, which places it comfortably in the CSPN lane, the latter may be bluer than the strip. EGB 1 (which is a PN mimic, but whose central star is a hot and young WD, likely only slightly more evolved than a typical CSPN) is within the CSPN lane, and finally, DeHt 5, which is also a PN mimic, but likely a post-RGB star, also resides in the CSPN lane. The SEDs of CSPN disks are also compared to those of the debris disks around cool WDs in Figure 5; the WD SEDs are flatter (hotter) and overall about 100 times dimmer.

We also note the disks around post-AGB stars with no nebula (van Winckel 2003) and those with a pre-PN (Section 4), which are also plotted in Figure 4. The plotted points for the naked post-AGB stars (with no nebula) are averages of approximately 10 objects with an oxygen-rich chemistry and 10 objects with 


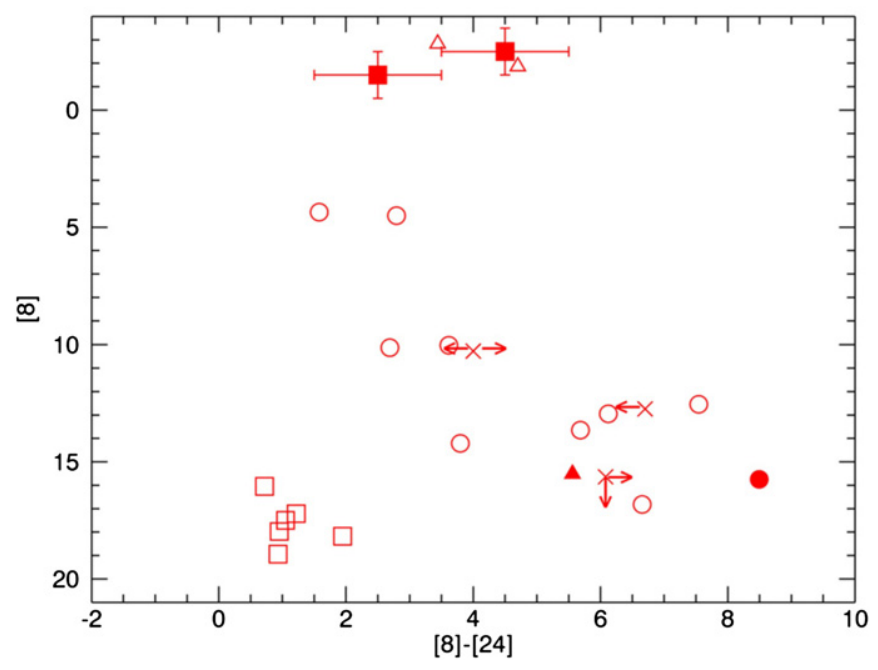

Figure 4. Color-magnitude diagram ([8-24] vs. [8]) plotting IRAC $8 \mu \mathrm{m}$ magnitude against the color, IRAC $8 \mu$ m magnitude - MIPS $24 \mu$ m magnitude. It shows the average position of 10 LMC post-AGB stars with carbon-rich chemistry and 10 with oxygen-rich chemistry (filled squares; Woods et al. 2011), GD 56, GD 133, and 4 other WDs with disks (open squares; Jura et al. 2007a), the central stars of the Helix PN (filled circle; Su et al. 2007), DeHt 5, a post-RGB star (filled triangle), 8 CSPNe and 1 CSPN mimic (open circles; Bilíková et al. 2012), A21 (X + right and down arrows, it was not detected at $8 \mu \mathrm{m}$; Bilíková et al. 2012), NGC 246 (X + left arrow, it was not detected at $24 \mu \mathrm{m}$; Bilíková et al. 2012), and NGC 7139 (X + left and right arrows, it was not observed at $24 \mu \mathrm{m}$; Bilíková et al. 2012). All fluxes have been scaled to $500 \mathrm{pc}$ and converted to magnitudes.

(A color version of this figure is available in the online journal.)

a carbon-rich chemistry (Woods et al. 2011). The error bars indicate the range of the individual values. For the pre-PN group, we use the NIR measurements from Bright et al. (2012) and S. N. Bright et al. (2014, in preparation), extracted from interferometric observations that measured the small disk rather than the overall dusty pre-PN. All post-AGB disks, whether a pre-PN is present or not, cluster together. Post-AGB disks are brighter than all other disks. Their colors are intermediate between the hot WD debris disks and the cooler CSPN dim disks and similar to the colors of the brighter, hotter CSPN group. All post-AGB stars, whether with a pre-PN or not, are stars which are close to the AGB (temperatures of a few thousand Kelvin; Table 2). Their disks either just formed (as may be the case for the central stars of pre-PN) or even if they are older (as may be the case for the post-AGB with no nebula whose evolution may have been stalled by accretion; van Winckel et al. 2009), their Keplerian nature ensures that they did not expand away (Bright et al. 2012; S. N. Bright et al. 2014, in preparation).

We speculate here that the warm, bright disks around postAGB stars, objects which have just left the AGB, become dim, red ones as the disk expands and disperses, and the star turns the "knee" of the HR diagram and dims. Hotter central stars would have cooler disks if the hotter and dimmer star is destroying the nearby dust and the bulk of the emission is overall farther from the center of radiation.

We also notice that almost all the CSPNe with 8 or $24 \mu \mathrm{m}$ point source detections appear to be binaries or binary candidates. NGC 246, EGB 6, and K 1-22 are wide binaries (where EGB 6 has a known disk around the companion; Bond \& Ciardullo 1999; Liebert et al. 2013; Ciardullo et al. 1999); NGC 2438 has a detected infrared excess consistent with an M3 V companion (although it could also be due to a warm disk Bilíková et al. 2012). DeHt 5, EGB 1, and NGC 6853 have $J$-band flux excesses consistent with companions with spectral type M5 V (De Marco et al. 2013), Sh 2-188 has a low- $\sigma$ detection of an I-band excess consistent with a companion of spectral type M4 V (De Marco et al. 2013). NGC 2346 is a close, single-lined spectroscopic binary with an A5 V companion to a hot star (Mendez \& Niemela 1981; Smalley 1997). The only objects in our sample that are not at present known to be binaries are NGC 6804,
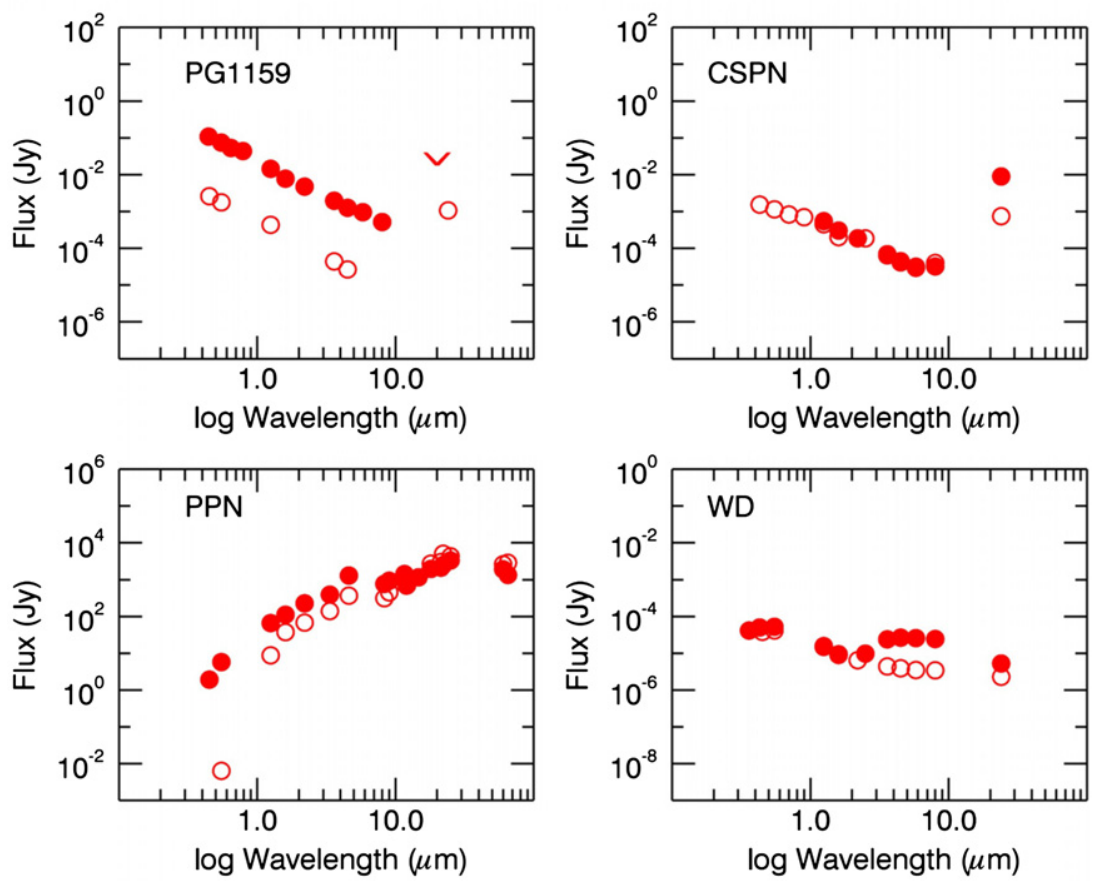

Figure 5. Top left: SEDs for NGC 246 (filled) and A21 (open). Top right: SEDs for the central star of the Helix nebula (filled) and DeHt5 (open). Bottom left: SEDs for IRAS 08005-2356 (filled) and IRAS 16279-4757 (open). Bottom right: SEDs for GD 56 (filled) and GD 133 (open) All fluxes have been scaled to 500 pc.

(A color version of this figure is available in the online journal.) 
A21, Sh 2-216, NGC 7139, and the Helix central star; the Helix central star has been thoroughly investigated and no companion has been detected down to a spectral type of mid-to-late $\mathrm{M}(\mathrm{Su}$ et al. 2007). The lack of a detected companion does not prove the lack of a companion. However, we cannot at this time state that all CSPNe with disks are binaries. Detecting fainter and fainter companions as well as more disks will allow us to corroborate or dismiss an association between binarity and disks. It is interesting to note that no cool WD with a dusty disk is known to have a close binary companion. One such WD binary (Debes et al. 2012), which was suggested to have a disk, seems instead to be magnetic (Parsons et al. 2013).

Finally, the low estimate of the dust mass of the Helix disk (Su et al. 2007) is about the same as that of the solar system's Kuiper belt $\left(\sim 3 \times 10^{-7} M_{\odot}\right.$; Su et al. 2007), is 1000 times lower than the mass of the disk around the [WC10] CSPN CPD $-56^{\circ} 8032$ (Cohen et al. 1999; De Marco et al. 2002; $6 \times 10^{-4} M_{\odot}$; an average of the two estimates of Cohen et al. 1999 and Clayton et al. 2011) and is also much less massive than the post-AGB disks for which we have data $\left(10^{-5}\right.$ to $10^{-3} M_{\odot}$; S. N. Bright 2014, in preparation). Disk dust masses determined for 11 disks around central stars of bona fide PNe by J. Bilíková ( $\mathrm{PhD}$ thesis and in preparation) also show high values once all dust components are included.

\section{CONCLUSIONS}

Dusty disks have been detected around about $18 \%$ of CSPNe. One out of five PG 1159 stars with PNe (Chu et al. 2011; Bilíková et al. 2012) show evidence of a disk, in line with the rest of the PN group. This may indicate that PG 1159 stars do not show disks more often than non-PG 1159 CSPNe. On the other hand, the statistics of PG 1195 CSPNe are extremely weak. It is interesting that none of the PG 1159 stars, which have no $\mathrm{PNe}$ and are therefore $10^{4}-10^{5} \mathrm{yr}$ older, shows evidence for a dusty disk.

The characteristics of the disks, such as mass, radius, composition, and temperature will yield their origin. Currently, however, we have only partial information on disks around a range of object classes, which makes it difficult to draw accurate conclusions. While the rare dust disks found around old DZ WDs are certainly of a different nature from those around CSPNe, it is still unclear what the nature of the CSPN disks is. Do they derive from the pulverization of KBOs, as is plausible for the Helix PN, or are they dust formed in the ejecta of AGB stars? Based on the colors and brightness of the CSPN disks relative to those of stars that have just left the AGB, one may argue that the origin of CSPN disks is from AGB ejecta.

One may also argue that CSPN disks may themselves have more than one origin. Disks around hydrogen-deficient [WC] and PG 1159 CSPN could derive from a different evolution from those around hydrogen-normal CSPNe and cooler postAGB stars (with or without PNe). On the other hand, on the color-magnitude diagram, the Helix CSPN disk resides close to other hot CSPNe, possibly indicating a common origin.

Finally, it came as a surprise that 8 out of 13 CSPN with disks detected because of 8 or $24 \mu \mathrm{m}$ excess are either binaries or likely/possible binaries. This connection, which should be further explored, may argue for an AGB origin.

This work was supported by Spitzer Space Telescope RSA No. 1364990 issued by Caltech/JPL. J.N. is supported by NSF AAP Fellowship AST-1102738 and by NASA HST grant AR-12146.04-A. O.D. gratefully acknowledges support from the Australian Research Council Future Fellowship grant FT120100452. T.R. is supported by the German Aerospace Center (DLR, grant 05 OR 1301).

\section{REFERENCES}

Aannestad, P. A., Kenyon, S. J., Hammond, G. L., \& Sion, E. M. 1993, AJ, 105,1033

Abazajian, K. N., Adelman-McCarthy, J. K., Agüeros, M. A., et al. 2009, ApJS, 182,543

Bannister, N. P., Barstow, M. A., Holberg, J. B., \& Bruhweiler, F. C. 2003, MNRAS, 341, 477

Barber, S. D., Patterson, A. J., Kilic, M., et al. 2012, ApJ, 760, 26

Bilíková, J., Chu, Y.-H., Gruendl, R. A., Su, K. Y. L., \& De Marco, O. 2012, ApJS, 200, 3

Bond, H. E., \& Ciardullo, R. 1999, PASP, 111, 217

Bright, S. N., De Marco, O., Chesneau, O., et al. 2012, in IAU Symp. 283, Planetary Nebulae: An Eye to the Future, ed. A. Manchado, L. Stanghellini, \& D. Schönberner (Cambridge: Cambridge Univ. Press), 115

Brinkworth, C. S., Gänsicke, B. T., Girven, J. M., et al. 2012, ApJ, 750, 86

Brinkworth, C. S., Gänsicke, B. T., Marsh, T. R., Hoard, D. W., \& Tappert, C. 2009, ApJ, 696, 1402

Chu, Y.-H., Su, K. Y. L., Bilikova, J., et al. 2011, AJ, 142, 75

Ciardullo, R., Bond, H. E., Sipior, M. S., et al. 1999, AJ, 118, 488

Clayton, G. C., De Marco, O., Whitney, B. A., et al. 2011, AJ, 142, 54

Cohen, M., Barlow, M. J., Sylvester, R. J., et al. 1999, ApJL, 513, L135

Cohen, M., Megeath, S. T., Hammersley, P. L., Martín-Luis, F., \& Stauffer, J. 2003, AJ, 125, 2645

Debes, J. H., Hoard, D. W., Farihi, J., et al. 2012, ApJ, 759, 37

De Marco, O., Barlow, M. J., \& Cohen, M. 2002, ApJL, 574, L83

De Marco, O., Passy, J.-C., Frew, D. J., Moe, M., \& Jacoby, G. H. 2013, MNRAS, 428, 2118

De Marco, O., \& Soker, N. 2002, PASP, 114, 602

Dong, R., Wang, Y., Lin, D. N. C., \& Liu, X.-W. 2010, ApJ, 715, 1036

Dreizler, S., \& Heber, U. 1998, A\&A, 334, 618

Dupuis, J., Fontaine, G., Pelletier, C., \& Wesemael, F. 1993, ApJS, 84, 73

Farihi, J., Jura, M., \& Zuckerman, B. 2009, ApJ, 694, 805

Frew, D. J. 2008, PhD thesis, Macquarie Univ.

Frew, D. J., \& Parker, Q. A. 2010, PASA, 27, 129

Furlan, E., Hartmann, L., Calvet, N., et al. 2006, ApJS, 165, 568

Gänsicke, B. T., Koester, D., Farihi, J., et al. 2012, MNRAS, 424, 333

Gänsicke, B. T., Marsh, T. R., Southworth, J., \& Rebassa-Mansergas, A. 2006, Sci, 314, 1908

Geier, S., Napiwotzki, R., Heber, U., \& Nelemans, G. 2011, A\&A, 528, L16

Gianninas, A., Bergeron, P., Dupuis, J., \& Ruiz, M. T. 2010, ApJ, 720, 581

Girven, J., Gänsicke, B. T., Steeghs, D., \& Koester, D. 2011, MNRAS, 417,1210

Graham, J. R., Matthews, K., Neugebauer, G., \& Soifer, B. T. 1990, ApJ, 357,216

Guiles, S., Bernard-Salas, J., Pottasch, S. R., \& Roellig, T. L. 2007, ApJ, 660,1282

Higdon, S. J. U., Devost, D., Higdon, J. L., et al. 2004, PASP, 116, 975

Hodge, T. M., Kraemer, K. E., Price, S. D., \& Walker, H. J. 2004, ApJS, 151,299

Houck, J. R., Roellig, T. L., van Cleve, J., et al. 2004, ApJS, 154, 18

Hu, J. Y., Slijkhuis, S., de Jong, T., \& Jiang, B. W. 1993, A\&AS, 100, 413

Jura, M. 2003, ApJL, 584, L91

Jura, M., Farihi, J., \& Zuckerman, B. 2007a, ApJ, 663, 1285

Jura, M., Farihi, J., Zuckerman, B., \& Becklin, E. E. 2007b, AJ, 133, 1927

Jura, M., Xu, S., Klein, B., Koester, D., \& Zuckerman, B. 2012, ApJ, 750, 69

Kato, T., Nogami, D., \& Baba, H. 2001, PASJ, 53, 901

Kilkenny, D., van Wyk, F., Roberts, G., Marang, F., \& Cooper, D. 1998, MNRAS, 294, 93

Koesterke, L. 2001, Ap\&SS, 275, 41

Koornneef, J., \& Pottasch, S. R. 1998, A\&A, 335, 277

Liebert, J., Bond, H. E., Dufour, P., et al. 2013, ApJ, 769, 32

Matsuura, M., Zijlstra, A. A., Molster, F. J., et al. 2004, ApJ, 604, 791

Mendez, R. H., \& Niemela, V. S. 1981, ApJ, 250, 240

Napiwotzki, R. 1999, A\&A, 350, 101

Parsons, S. G., Marsh, T. R., Gänsicke, B. T., et al. 2013, MNRAS, 436, 241

Perea-Calderón, J. V., García-Hernández, D. A., García-Lario, P., Szczerba, R., \& Bobrowsky, M. 2009, A\&A, 495, L5

Phillips, J. P. 2003, MNRAS, 344, 501

Reach, W. T., Kuchner, M. J., von Hippel, T., et al. 2005, ApJL, 635, L161

Sargent, B., Forrest, W. J., D’Alessio, P., et al. 2006, ApJ, 645, 395

Sargent, B. A., Forrest, W. J., Tayrien, C., et al. 2009, ApJ, 690, 1193 
Slijkhuis, S., de Jong, T., \& Hu, J. Y. 1991, A\&A, 248, 547

Smalley, B. 1997, Obs, 117, 338

Steele, P. R., Burleigh, M. R., Dobbie, P. D., et al. 2011, MNRAS, 416, 2768

Su, K. Y. L., Chu, Y.-H., Rieke, G. H., et al. 2007, ApJL, 657, L41

Tweedy, R. W., \& Kwitter, K. B. 1994, ApJL, 433, L93

van Winckel, H. 2003, ARA\&A, 41, 391

van Winckel, H., Lloyd Evans, T., Briquet, M., et al. 2009, A\&A, 505, 1221

Vassiliadis, E., \& Wood, P. R. 1993, ApJ, 413, 641

Watson, D. M., Bohac, C. J., Hull, C., et al. 2007, Natur, 448, 1026
Weidemann, V. 1960, ApJ, 131, 638

Werner, K., Bagschik, K., Rauch, T., \& Napiwotzki, R. 1997, A\&A, 327,721

Werner, K., \& Herwig, F. 2006, PASP, 118, 183

Werner, K., Rauch, T., Reiff, E., Kruk, J. W., \& Napiwotzki, R. 2004, A\&A, 427, 685

Woods, P. M., Oliveira, J. M., Kemper, F., et al. 2011, MNRAS, 411, 1597

Xu, S., \& Jura, M. 2012, ApJ, 745, 88

Zuckerman, B., \& Becklin, E. E. 1987, Natur, 330, 138 